\title{
Editorial: Singular and Correlation Optics
}

\author{
Oleg V. Angelsky ${ }^{1,2}$, Aleksandr Y. Bekshaev ${ }^{3 *}$, Steen G. Hanson ${ }^{4}$, Igor I. Mokhun ${ }^{2}$, \\ Mikhail V. Vasnetsov ${ }^{5}$ and Wei Wang ${ }^{6}$ \\ ${ }^{1}$ Research Institute of Zhejiang University-Taizhou, Taizhou, China, ${ }^{2}$ Correlation Optics Department, College of Engineering, \\ Chernivtsi National University, Chernivtsi, Ukraine, ${ }^{3}$ Physics Research Institute, Odessa I.I. Mechnikov National University, \\ Odessa, Ukraine, ${ }^{4}$ DTU Fotonik, Department of Photonics Engineering, Roskilde, Denmark, ${ }^{5}$ Institute of Physics, National \\ Academy of Sciences of Ukraine (NAN Ukraine), Kyiv, Ukraine, ${ }^{6}$ Heriot-Watt University Edinburgh, Edinburgh, United Kingdom
}

Keywords: structured light, singular optics, correlation optics, optical vortex, angular momentum

\section{Editorial on the Research Topic}

\section{Singular and Correlation Optics}

Singularities in wave fields form a relatively young concept of wave physics originating from the seminal article by Nye and Berry [1]. Initially, this concept seemed rather abstract and curious, but the development of laser optics has demonstrated the ubiquity of optical singularities and their exceptional power in characterization, control, and applications of light fields. The era of singular optics started $[2,3]$, and now, it is difficult to indicate another area of optics that is developing so rapidly and steadily.

Different sorts of optical singularities, involving the phase, polarization, energy flow (Poynting singularities), constitute a new paradigm of physical optics. In generic fields, they form interconnected and coherent networks (singular skeletons) that unite point-like, linear and surface singularities. Due to natural correlation rules, each singularity "organizes" the field nearby, so the singular skeleton supplies efficient means for the characterization of the whole field, important for optical data processing and measurements. In these aspects, singular optics fruitfully harnesses the ideas and notions of correlation optics [4]. On the other hand, specific behavior of the optical field in the vicinity of a distinct singularity can be favorable for optical trapping, micromanipulation, sensitive metrology, and diagnostics. Importantly, singular networks possess a topological nature that makes them especially stable against disturbances and useful, for example, for optical data compression and transmission.

All the aspects mentioned (which hardly exhaust the list of impressive and useful features of singular light fields) are discussed in the present research topic. It includes six articles, of which four describe original research results and two offer detailed reviews. The review by Angelsky et al., "Structured Light: Ideas and Concepts" briefly presents some essential concepts and features of light fields with strong spatial inhomogeneity of amplitude, phase, polarization, and other parameters, which are the typical objects of singular optics. A special attention is paid to the field dynamical characteristics (energy, momentum, angular momentum, and their derivatives) which are considered not only as mechanical attributes of the field but also as its application-oriented descriptive parameters. The dynamical properties of paraxial beams and evanescent waves (including surface plasmon-polaritons) are analyzed in more detail, and applications of structured light fields for optical manipulation, metrology, probing, and data processing are described. The second review "Synthesized Vortex Beams in the Turbulent Atmosphere" presented by Aksenov et al., is devoted to a promising singular-optics application for optical communications. It summarizes the optical vortex beam formation through the combination of wave fields produced by a fiber array generating a set of sub-beams with controllable initial phases. In this 
way, the helical-phase "combined" beam appears with controllable orbital angular momentum (OAM). The encoding of information by the OAM value is demonstrated in laboratory experiments. Statistical characteristics of the vortex Laguerre-Gaussian and synthesized beams propagating through the turbulent atmosphere are studied theoretically, and their potential for the atmosphere-channel data transmission is evaluated.

The rest of contributions present original research results. The article "Toward a Corrected Knife-Edge-Based Reconstruction of Tightly Focused Higher Order Beams" by Orlov et al., analyzes specific difficulties of the singular-light beam profiling by the knife-edge method. The authors study possible distortions in the shape and position of the reconstructed beam profile due to the light beam interaction with the knife material and introduce corrections to the standard knife-edge evaluation method for highly focused radially and azimuthally polarized beams and their linearly polarized constituents. An adapted knife-edge reconstruction technique is presented, and proof-of-concept tests are shown.

The contribution by Ruchi and Paramasivam Senthilkumaran "Polarization Singularities and Intensity Degeneracies" investigates the typical, for singular and correlation optics, effects of interplay between the phase, amplitude, and polarization distributions. In particular, fringes with high contrast occur when the interfering beams are in the same state of polarization (SOP). But the authors show that superpositions of multiple beams in different SOPs form lattice patterns consisting of polarization singularities and with high intensity contrast. By changing the SOPs of the individual beams, same intensity distributions can be produced. These are termed as "degenerate intensity patterns" and can be achieved if the SOP changes follow certain rules. This study illustrates the limitations of intensity-based measurements in identifying polarization singularities.

Promising ideas on optical manipulation are presented in "Extraordinary Manifestation of Evanescent Wave in

\section{REFERENCES}

1. Nye JF, Berry MV. Dislocations in wave trains. Proc R Soc Lond A. Math Phys Sci (1974) 336(1605):165-90. doi:10.1098/rspa.1974.0012

2. Soskin MS, Vasnetsov MV. Singular optics. Prog Opt (2001) 42:219-76. doi:10. 1016/S0079-6638(01)80018-4

3. Dennis MR, O'Holleran K, Padgett MJ. Singular optics: optical vortices and polarization singularities. Prog Opt (2009) 53:293-363. doi:10.1016/S0079-6638(08)00205-9

4. Angelsky OV, Polyanskii PV, Felde CV. The emerging field of correlation optics. Opt Photon News (2012) 23(4):25-9. doi:10.1364/OPN.23.4.000025
Biomedical Application" by Angelsky et al. They study mechanical effects produced by evanescent waves on nanoobjects of inorganic and organic (in particular, formed blood elements) origin, suspended in a biological liquid. Especially, the action directed perpendicular to the Poynting vector is of particular interest. The evanescent wave is formed due to the total internal reflection realized at the interface between a glass prism and a biological medium, and its control is performed by changing the azimuth of the linearly polarized incident plane wave. The article summarizes the latest theoretical and experimental results illustrating the rectilinear and rotational motions of investigated objects in a biological environment. This research illustrates new possibilities in the optical control of the nano-objects and their possible prospects in biology, medicine, pharmacology, and precision chemistry.

Important issues associated with "Singularities of Partially Polarized Vortex Beams" are discussed by Raburn and Gbur. They notice that singularities in the polarization state of nonuniform electromagnetic beams have been a topic of interest for many years, as have singularities in the correlation functions of random scalar wave fields. However, the intersection of these phenomena, namely, singularities in the polarization state of partially coherent wave fields, is not explored properly. The authors use a simple model of a partially coherent electromagnetic vortex beam to highlight three different ways that one can define polarization singularities in scalar wave fields, one of which has not been previously discussed.

We hope that the current research topic collection supplies a useful exhibition of the newest results and ideas relating to singular and correlation optics, which will promote further progress in this blooming branch of physical science.

\section{AUTHOR CONTRIBUTIONS}

All authors listed have made a substantial, direct, and intellectual contribution to the work and approved it for publication.

Conflict of Interest: The authors declare that the research was conducted in the absence of any commercial or financial relationships that could be construed as a potential conflict of interest.

Copyright (c) 2021 Angelsky, Bekshaev, Hanson, Mokhun, Vasnetsov and Wang. This is an open-access article distributed under the terms of the Creative Commons Attribution License (CC BY). The use, distribution or reproduction in other forums is permitted, provided the original author(s) and the copyright owner(s) are credited and that the original publication in this journal is cited, in accordance with accepted academic practice. No use, distribution or reproduction is permitted which does not comply with these terms. 